\title{
Coronavirus (COVID-19) - Kenyan case: A Review onprioritizing immunonutrition in Prevention and Management
}

\author{
Dr. Carren Adhiambo Otieno
}

\begin{abstract}
At the end of the year 2019, a novel virus, severe acute respiratory syndrome coronavirus 2(SARS-CoV-2), causing severe acute respiratory syndrome expanded globally fromWuhan, China.The beginning of 2020 saw the emergence of COVID-19 outbreak which was in March 2020 declared a global pandemic by the World Health Organization (WHO). Coronaviruses infect the upper gastrointestinal and respiratory tractof the mammals including humans and birds. Animals rarely spread coronaviruses to humans but it subsequently spreads between humans via respiratory droplets produced when a person sneezes or coughs.Despite great efforts globally, there is no specific antiviral treatment and vaccinecurrently recommended for COVID-19-19. However, prevention and management are the best options. Using research reports, online databases, WHO guidelines and recent articles on COVID-19, i carried out systematic review of immunity and nutrition in the prevention and management of the disease. This article may be useful in creating awareness among the public, to combat COVID-19through balanced nutrition.
\end{abstract}

Index Terms - COVID-19-2019,immunityprevention, Respiratory syndrome, SARS.

\section{INTRODUCTION}

The recent global outbreak of COVID-19 imposed catastrophic impacts on all age groups,specifically among elderly populations and those with underlying health conditions. Currently no treatment or vaccine has been produced which has led many researchers finding out approaches that can reduce the number of severe cases and consequently reduce mortality. A study is necessary so as to better understand the defense mechanisms of the immune system against COVID-19 and to develop effective management and treatments of the viral infection (Daneshkhah et al., 2020).

In the initial stages of the Novel coronavirus 2019 (COVID-19) pandemic, Sub-Saharan Africa reported the lowest infection rates. From March 2020, the spread had been increasing steadily. According to Johns Hopkins University, by July 2020, Global death toll had surged past 592,977 with over 13, 960,499 million confirmed cases. Recoveries globally by July, 2020 was $8,293,613$. In Africa, there had been more than 644,333 confirmed cases, 14,047 deaths and 334,978 recoveries. According to CGTN Africa, Kenya continues to battle with the COVID-19 pandemic with cases rising each day making it more than a health crisis.It is a human, economic and social crisis as it attacks societies at their core.In Kenya, by July, 2020 COVID-19 confirmed cases had reached 11,673.Severe Acute Respiratory Syndrome Coronavirus2 (SARS-CoV-2) belongs to the Sarbecovirus sub-genus of the Coronaviridae family, and is the seventh coronavirus known to infect humans.

Coronaviruses are a large family of enveloped RNA viruses, some of which cause illness in people such as common cold, SARS, MERS and others that circulate among mammals like bats, camels and birds. Similar to SARS and MERS, it is thought that human transmission occurs via respiratory droplets produced when a person sneezes or coughs. Coronavirus 2019 (COVID-19-19) has a typical crown-like appearance under an electron microscope due to the presence of glycoprotein spikes on its envelope (Gennaro et al, 2020). There is an imminent need to better understand this new virus and to develop ways to control its spread.

Currently, the efforts against COVID-19 consists of supportive therapy with recommended preventive measures such as washing your hands with soap, covering the mouth when coughing, maintaining 1-meter distance from other people and monitoring and self-isolation for fourteen days for people who suspect they are infected (Hafeez et al., 2020).On top of basic illness, prevention and real defense against disease is a strong immune system. (Hafeez et al, 2020). In the light of the current pandemic of COVID-19, there is need to evaluate the evidence on enhancing immunity in viral infections. This review mainly focuses on, coronavirus. Practical recommendations have been drawn on both preventive and therapeutic nutritional interventions for COVID-19.There is adequate evidence to demonstrate that immune response is weakened by inadequate nutrition (COVID-19 UNICEF Programme 2020). Verification of the nutritional status of COVID-19-19 infected patients before, during and after the administration of general treatment is necessary. Providing appropriate nutrition together with use of supplemental diet as the base for nutrition management is paramount. Most people tend to be skeptical of the benefits of dietary supplements and neutraceuticals yet pharmaceutical companies produce very expensive drugs and sometimes ineffective. Trials have been done on use of sweetable and cheaper supplements particularly on the immune suppressedindividuals

(https://link.springer.com/article/10.1186/s12974-017-1051).

COVID-19 is particularly detrimental to members of those social groups in most vulnerable situations, living in poverty, 
older persons, persons with disabilities, youths and the homeless. These people stand to suffer disproportionately both from the pandemic and its aftermath due to limited movement, fewer employment opportunities and increased anxiety. If the social crisis is not addressed, COVID-19 pandemic can overwhelm the economy as emphasized by the United Nations secretary general during the launch of a COVID-19global humanitarian response plan that we must come to the aid of the vulnerable in order to combat the virus (UN).

Nairobi's more than 4 million residents reside in urban slums. Characterized by high population density, small informal dwellings, lack of access to clean water, multi-generational households, shared sanitation facilities among multiple households, a high level of both inter and intra-social mixing within slums(https://www.who.int/bulletin/online_first/20-260281. pdf).Most of the slum residents experience poor health outcomes related to both poor environmental conditions and inability to pay for medical care so they have a higher overall mortality and mobidity rate. The population is therefore vulnerable to economic shocks, as most of them rely on income from the informal sector. Disease outbreaks in past pandemics for example the ebola have been accelerated in slum settings. This necessitates stringent preventive measures in such like places (Austin et al, 2020).

There is no specific treatment for the new Coronavirus (COVID-19) which is not unusual as there are no particular treatments for most viruses. They need to run their course, allowing the body to make its antibodies to counter the virus. There is no present vaccine for the new form of COVID-19 virus. Prescribed antibiotics used to treat bacterial or fungal infections have proved non- effective against it. The most reliable, cheap and effective way to manage or prevent the COVID-19is to boost immune system. Nutritional status should be maintained with appropriate intake of calories, vitamins, minerals and water that should be continuously provided by a healthy diet with supplements where necessary. There is therefore need to assess the nutritional status of COVID-19-infected patient, those in quarantine and the vulnerable groups on the basis of management. However, prevention measures remain the first priority and strategy together with proper hygiene and staying home.

\section{ORIGIN, TAXONOMY AND MOLECULAR ASPECTS OF COVID-19}

There are four genera of CoVs: coronavirus(alpha $\mathrm{CoV}$ ), coronavirus (betaCoV) probably found in bats and rodents, while coronavirus(delta $\mathrm{CoV}$ ) and coronavirus (gammaCoV) present in avian species (Gennaro et al, 2020). The virus has a natural and zoonotic origin; the natural selection in an animal host before zoonotic transfer and natural selection in humans following zoonotic transfer (Gennaro et al, 2020). The genome of this coronavirus resembles the SARS-CoV and MERS-CoV (Lu et al., 2020; Renet al., 2020). Both MERS-CoV and SARSCoV have their origin in the bats (Cui et al., 2019).Infection begins when the virus enters the host cell, the virus particle is uncoated and the spike protein attaches to its complementary host cell receptor. The main target of coronavirus is lungs. The virus spikes getattached to the cell receptors of the lungs known as angiotensin-converting enzyme 2 (ACE2) receptors (Jaimes et al., 2020; Wanet al., 2020). Proteolytic cleavage occurs at SARS-CoV S protein at position (S2') (Belouzard et al., 2009). After attachment, a proteolytic enzyme of the host cell cleaves and activates the receptor attached spike macromolecule. The chemical structure of COVID-19 RNA consists of $5^{\prime}$ methylated head and a $3^{\prime}$ polyadenylated tail throughwhich the RNA attaches to the free ribosomes of the host cell. This leads to the process of translation and formation of a long polypeptide chain. This protein has an enzyme- Proteases which break the polyprotein (Hafeez et al., 2020). SARS-CoV-2 propagate through RNA replication using RNA-dependent RNA polymerases enzyme. This virus is capable of mutating, posing a challenge for treatment and control. (Li et al., 2020), (Wan et al.,2020). The pathological conditions of coronavirus include greater counts of chemokines, cytokines, leukocytes, high levels of plasma pro-inflammatory cytokines and $\mathrm{C}$ - reactive protein. (Carlos et al., 2020).

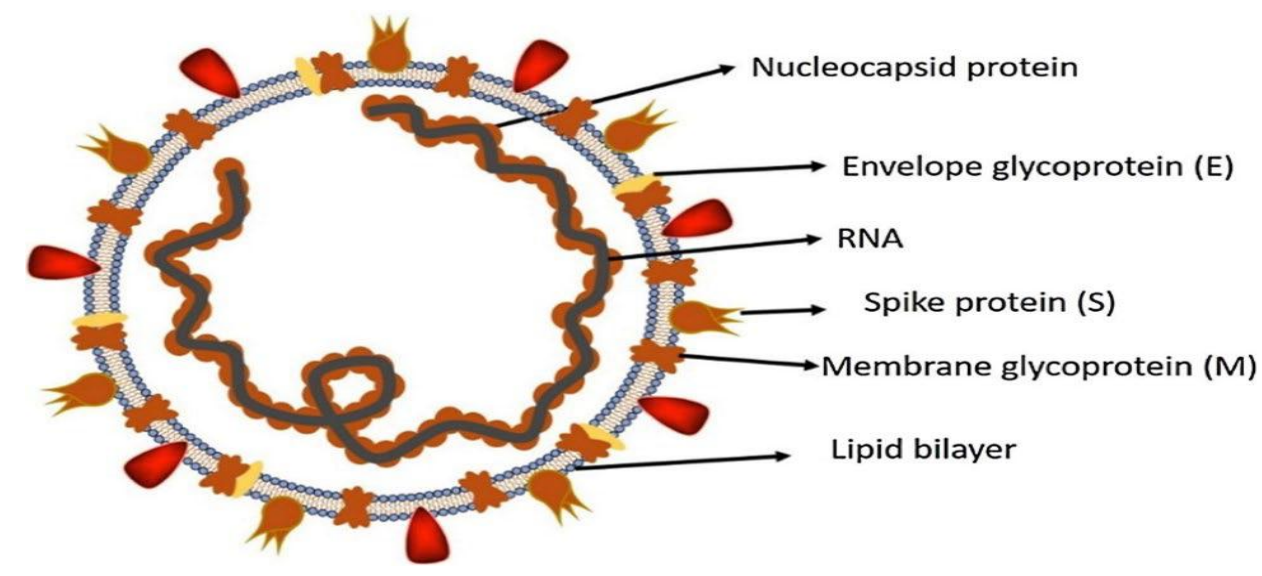

Hafeez et al., 2020 


\section{COVID-19 DISEASE SYMPTOMS}

Coronaviruses infect the upper gastrointestinal and respiratory tract of mammals including humans and birds. These viruses cause many diseases in animals and human beings but this paper is limited to SARS-CoV-2, leading to COVID-19-19 disease. The period from the onset of symptoms to death ranges from 6 to 41 days with amedian of 14 days. This period is dependent on the age and status of the patient'simmune system. The most common symptoms at onset of COVID-19 illness are fever, cough, and fatigueor muscle pain, sneezing, sore throat, dry cough. Other symptoms include respiratory problems, sputum production, headache, haemoptysis, diarrhoea, dyspnoea, and lymphopenia (Gennaro et al, 2020).Some severe cases include pneumonia, serious respiratory syndrome, septic shock, kidney failure and even death (Huang et al., 2020; Hui et al., 2020; Ren et al., 2020). Comorbidities such as diabetes, chronic obstructivepulmonary disease (COPD), lung diseases, heart diseases, cancer,hypertension and obesity are thought to increase disease severity (Gennaro et al., 2020). A percentage of COVID-19 patients often develop acute respiratory distress syndrome and acute lung injury (ARDS/ALI). The uncontrolled progressive inflammation in the lungs causes acute diffuse alveolar damage recognized as areas with ground glass opacities and other areas with increased density but without any recognizable vessels. As ARDS progress to the acute phase, alveolar flooding (edema), interstitial inflammation and compression steleclatis as well as increase in lung tissue and reduction in lung gas are observed. At this stage, COVID-19 patients with ARDS/ALI often require intubation and invasive mechanical ventilation to assist in breathing because of increased hypoxemic respiratory failure which results in acute diffuse alveolar damage. ARDS (Acute respiratory distress syndrome) and ALI (Acute lung injury) are often characterized by the accumulation of neutrophils in the lungs and increased production of inflammatory cytokines, chemokines, proteases and oxidants (https://pubmed.ncbi.nlm.nih.gov/15649738-melatonin-as-ahypnotic-pro/).

The nutritional recommendations for mild symptoms such as runny nose, fever, cough,headache and sore throat include; adequate fluids, adequate energy-rich foods, meat, milk, legumes and pulses, fruits and vegetables. There should be supplementation with Vitamin C, zinc, Vitamin A, B6, D, E, iron, Folate, fiber and enough sleep, reduced stress and enough exercise. Alcohol and tobacco products should be avoided. Coughs can be relieved by use of honey, pineapple and chicken soup. Sore throat can be relieved by taking tea, honey, ginger and turmeric. Culinary herbs like oregano, sage and cinnamon are able to improve antioxidant levels in the body. There should be limited intake of refined carbohydrates such as sugar, sweets, cake, soft drinks, sugar sweetened beverages, foods containing trans-fats and saturated fats e.g. fat and skin from meat, hydrogenated vegetable oils, fried foods, cookies, and pastries. (Kenya, clinical nutrition and dietetics reference manual). An array of micronutrients have been reported to be able to meet the complex needs of the immune system, including vitamins A, D, C, E, B6, B12, folate, copper, iron, zinc and selenium. Of the evidence, however, it was concluded that the largest body of evidence related to immune function existed for vitamin C, D and zinc(Hemilia, 2017).

\section{MODES OF TRANSMISSION}

Although coronaviruses are common in bats, no direct animal source of the epidemic has been identified to date. It is necessary to identify the intermediate species to stop the current spread and to prevent future human SARS-related coronavirus epidemics. Individuals with SARS-CoV-2 may remain asymptomatic for 2 to 14 days with an average of five dayspost-infection and some individuals likely transmit the virus without developing disease symptoms (Miguel, 2019). The coronavirus is spread by the sneezing, cough droplets and contact. It is considered most contagious when people are symptomatic, although transmission may be possible by asymptomatic patients (Hafeez et al, 2020). Normally, the virus enters the body through the mouth, nose and eyes (Transmission of Novel Coronavirus (2019-nCoV), 2020). The virus may infect a person at a distance of about $2 \mathrm{~m}$ radius and survive for $2 \mathrm{~h}$ to few days in cough and sneezing droplets lying on the surface or ground. An infection may be by touching an object or surface which has the virus. Other chances of the infection may arise if a person comes in contact with the infected person. 


\section{Transmission of COVID-19 to human host}

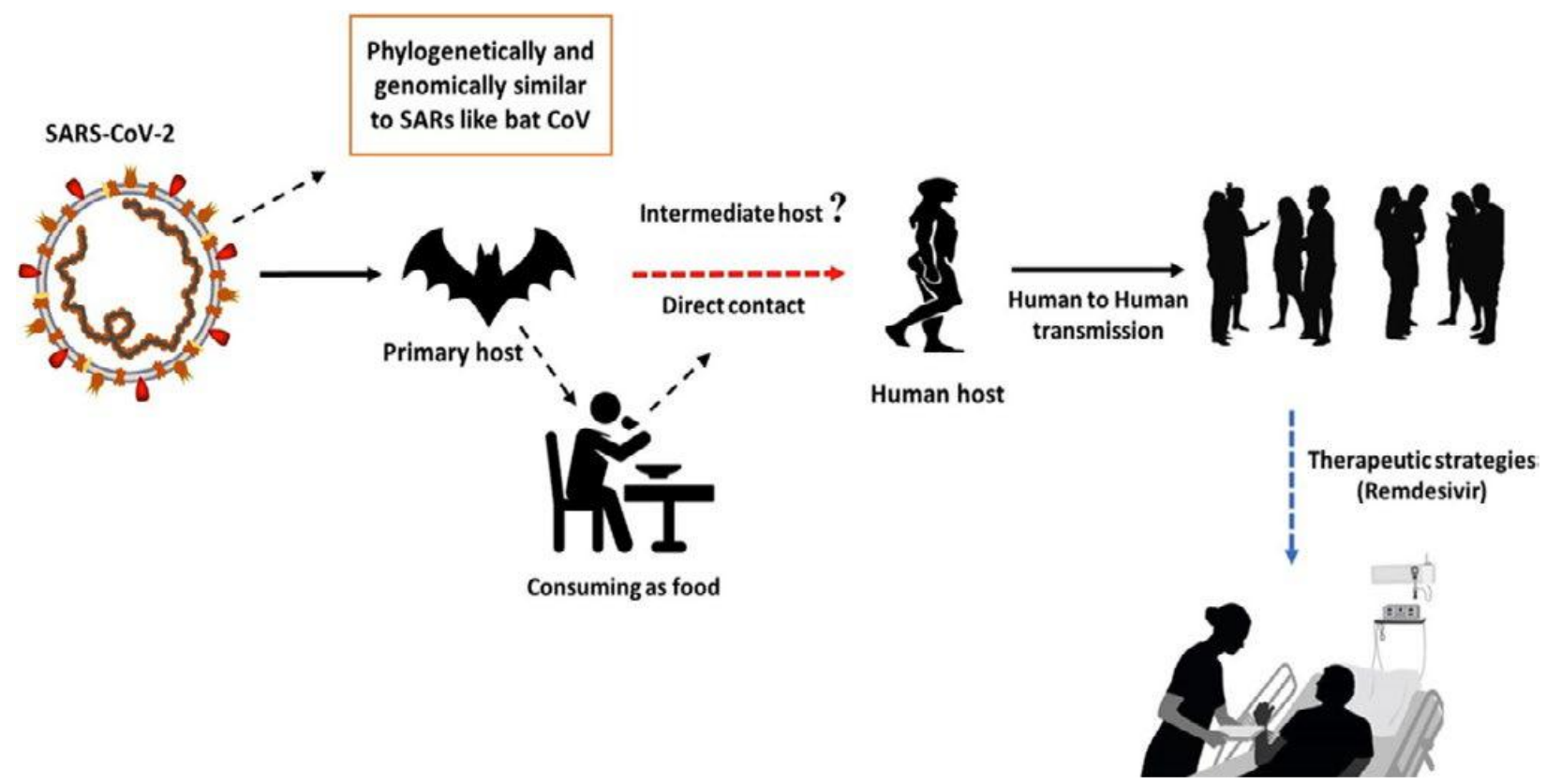

Hafeez et al, 2020

\section{DiagnOSIS OF COVID-19}

Diagnosis allows suspected people to know whether they are infected or not so as to receivethe care they need and to take measures against infecting others. The Coronavirus disease-2019 tracking and diagnostic testing are in understanding epidemiology, informing case management, and in suppressing transmission. To test for COVID-19, doctor or health practitioner may take samples, including a sample of saliva ,sputum, nasal swab and a throat swab, send to a laboratory for testing or follow the directions of local health authority ((Hafeez et al., 2020). Test for COVID-19 can be based on individual signs and symptoms. The doctor may also consider whether an individual had close contact with someone diagnosed with COVID-19 or travelled to or lived in any areas with ongoing community spread of COVID-19-19 within the last 14 days. The standard tool of diagnosis is by reverse transcriptionpolymerase chain reaction (rRT-PCR) from a throat swab or nasopharyngeal swab.The test looks for viral DNA in a sputum sample and can detect the virus earlier in the infection, butis quite time consuming. (Hafeez et al., 2020).A test may also be used that screens patient's blood samples for antibodies against the virus.It can identify the SARS-CoV-2 virus and includes methods that detect the presence of virus itself like RT-PCR, isothermal nucleic acid amplification and antigen. (Jennifer, 2020). Detection of antibodies (serological tests) can be used both for diagnosis and population surveillance. Antibody tests show how many people have had the disease, including those whose symptoms are still minor or who were asymptomatic. However, the duration and effectiveness of this immune response are still unclear and its accuracy is still questionable becausein some instances, antibodiesare often not detectable until a few days after symptoms begin.

\section{TREATMENT OF SARS - COV -2}

Treatment is essentially supportive. Role of antiviral agents is yet to be established. Treatment can be considered pharmacological with potential clinical benefit, adjunctive pharmacological treatments or pharmacological treatments where risks outweigh benefits. Control measures are needed for patients with high fever.Antipyretic drug treatment should be performed in case the temperature exceeds $38.5^{\circ} \mathrm{C}$. So far, the most promising compound for treating COVID-19 is the antiviral, remdesivir. It is currently in clinical trials for treating Ebola virus infections (Miguel, 2019). Remdesivir is a faltering monophosphoramidate prodrug of an adenosine equivalent that was developed by Gilead Sciences, Inc. in response to the Ebola outbreak in West Africa from 2014-2016.

Remdesivir was recently tested in a non-human primate model of MERS-CoV infection. Prophylactic treatment 24 hours prior to inoculation prevented MERS-CoV from causing clinical disease and inhibited viral replication in lung tissues, preventing formation of lung lesions. Initiation of treatment 12 hours after virus inoculation was similarly effective. Remdesivir has also shown effectiveness against a wide range of coronaviruses. Clinical trials for COVID-19 is necessary. In its active triphosphate nucleoside form, remdesivir binds to RNA-dependent RNA polymerase and acts as an RNA-chain terminator (Wang et al., 2020). It is highly selective for viral polymerases consequently projected to have a low predisposition to cause human toxicity. Sheahan (2020) confirmed a wide beneficial guide for remdesivir in a human airway epithelial cell model. (Sheahan et al., 2020). However much work needs to be done to gain a better understanding of the mechanics of COVID-19. Knowledge of howCOVID-19 interacts with the host ACE2 receptor should be explored. 
A great genetic obstruction to resistance in coronaviruses has been displayed by remdesivir with a long intracellular half-life for once daily dosing (Agostini et al., 2018, Siegel et al., 2017). For treatment of COVID-19, the dosage under trials is $200 \mathrm{mg}$ intravenously (IV) on day 1 followed by $100 \mathrm{mg}$ IV daily for up to 10 days, infused over 30-60 minutes. Developing clinical evidence and available in-vitro data suggest remdesivir is a promising agent for the treatment of COVID-19. More clinical trial enrollment and evidence by institutions should be done to investigate its use remdesivir for moderate-to-severe patients before they are recommended.

Chloroquine is a known antimalarial agent with anti-inflammatory and immunomodulatory activities. It has gained significant interest as a potential therapeutic option for the management of SARS- CoV- 2 (Wang et al., 2020). United States has had challenges of supply and cardiovascular toxicity which has set a limit barring the use of chloroquine.Hydroxychloroquine compound differs from chloroquine only by a single hydroxyl group and it is being considered as an alternative because of better tolerability than chloroquine favourable for rheumatological disorders. Very limited data has been made available to the public on the activity of hydroxychloroquine against coronaviruses.

Yao et al. (2020) performed a two-part study assessing the comparative in -vitro activity of chloroquine and hydroxychloroquine and demonstrated that the potency of hydroxychloroquine (EC50 of $0.72 \mu \mathrm{M}$ ) was greater than that of chloroquine (EC50 of $5.47 \mu \mathrm{M}$ ) against SARS-CoV-2 (Yao et al., 2020). Due to the unavailability of data on efficacy for hydroxychloroquine in treatment of SARS-CoV2 , thoughtful deliberation should be considered for effective dosing strategy.

Lopinavir is a human immunodeficiency virus 1 (HIV-1) protease inhibitor administered in fixed-dose combination with ritonavir (LPV/r), and it appears to block the main protease of SARS-CoV-1, inhibiting viral replication (Ratia et al., 2008). The antiviral activity of lopinavir against SARS-CoV-1 has been demonstrated with cells which are near the upper range of LPV plasma concentrations. This had been measured in patients with HIV(De Wilde et al., 2014, Lopez et al., 2011).

Real-world data for treatment of COVID-19 with LPV/r are emerging from Singapore (Young et al., 2020). Nevertheless, there are challenges in data interpretation especially in relation to affiliated drug therapies, time variation in therapy initiation, diverse severity of illness amongst SARS-CoV-2 patients, and the lack of standard treatments(Limet al., 2020; Han et al.,2020; Wang etal., 2020).

Nitazoxanide has proven potentin vitro activity against SARS CoV-2 (Wang et al., 2020). It exhibits broad spectrum in vitro antiviral activity against influenza, respiratory syncytial virus, parainfluenza, rotavirus, and norovirus amongst others in addition to coronaviruses due to mechanism of action based on interference with viral replication (Rossignol et al., 2016).

\section{A. Adjunctive pharmacological treatments}

Tocilizumab is a humanized monoclonal antibody that inhibits both membrane-bound and soluble interleukin-6 (IL-6) receptors. IL-6 is the main drivers of immunologic response and symptoms in patients with cytokine-release syndrome (CRS). The drug has gained importance in recent years for treatment of patients with CRS following chimeric antigen receptor Tcell (CAR T) therapy as a corticosteroid-sparing agent (Kotch et al., 2019). Hyper inflammatory states, cytokine storming as well as elevated IL-6, have been reported in severe SARS- CoV-2 patients. These were associated with increased death in patients in China (Zhou et al., 2020). Trials are ongoing in China evaluating safety and efficacy of tocilizumab for patients with COVID-19 pneumonia, but it is none registered in the United States. More data is expected regarding its efficacy because its' administration during the disease development is not yet defined, nor is there a known threshold of IL-6 for progression to severe disease.

Corticosteroids have been employed for the management of severe pneumonia due to coronaviruses. It has been useful in the management of the inflammatory effects presented in severe cases of the disease. Currently the data evaluating the role of corticosteroids as adjunctive care for severe coronavirus (SARS-CoV-1, MERS-CoV, and SARS-CoV-2) pneumonia are inconsistent, confusing, and inconclusive (Stockman et al., 2006). Decreased mortality in critically ill patients (Chen et al., 2006), longevity of time for viral clearance (Lee et al., 2004) or an increase in critical state of patients to death (Auyeung et al., 2005) has been confirmed.

There exists pharmacological treatments where risks outweigh benefits through use of agents like Ribavirin +/interferon with poor or inaccurate outcomes from the populations targeted e.g. hematologic toxicity (Booth et al., 2003, Lee et al., 2003). There exists agents ranging from pharmaceutical drugs to medicinal herbs currently under investigation for treatment and management of COVID-19.The only treatment of COVID-19now available includes symptomatic care and oxygen therapy.

\section{PREVENTION AND MANAGEMENT OF COVID-19}

The prevention and management are very important aspectsin COVID-19 control. People should take keen note on the latest information on COVID-19 outbreak provided by WHO and follow the directions of local health authority, prevent secondary infections and interrupt human-to-human transmission (Hafeez et al., 2020). The regular recommendations to minimize the infection are cleaning of one's area, hand cleaning with soap and sanitizer, mouth andnose coverage with mask during sneezing and coughing are essential. Thoroughly washing foodstuff before eating or cooking may help in this regard.The simple house-keeping disinfectants may kill the virus on the surfaces. Avoiding the interactionswith anyonesuspecting respiratory problems symptoms like sneezing, coughing, breathing problem should be adhered to. It is also advisable to stay at home if one has no much to do outside or maintaining an appropriate distance from people, refraining from touching eyes, nose, and mouth. 
Immunocompromised individuals are advised to avoid public gatherings. In case of symptoms, one should seek medical care early and follow advice given by healthcare provider (Gennaro et al., 2020). Preventive measures must focus on optimizing infection control protocols, self-isolation, and patient isolation during the provision of clinical care. The WHO advised against close contact with patients, farm animals, and wild animals (Cascella et al.,2020). Emergency medicine departments must apply strict hygiene measures for the control of infections. Healthcare personnel must use personal protective equipment such as N95 masks, FFP3 masks, gowns, eye protection, gloves, and gowns.

Patients with mild infections require early supportive management. This can be achieved by the use of acetaminophen, external cooling, oxygen therapy, nutritional supplements, and anti-bacterial therapy (Wang et al., 2020). Critically ill patients require high flow oxygen, extracorporeal membrane oxygenation (ECMO), glucocorticoid therapy, and convalescent plasma (Wang et al., 2020). (Cascella et al.,2020). Unnecessary administration of antibiotics should be avoided. (Ivory, 2017). Patients with respiratory failure may require intubation, mechanical ventilation, high-flow nasal oxygen, or non-invasive ventilation (Ivory, 2017).Treatment of septic shock requires hemodynamic support with the administration of vasopressors. Organ function support is necessary for patients with multiple organ dysfunction (Cascella et al.,2020).Therapeutically, aerosol administration of alpha-interferon (5 million units twice daily) (Wang et al., 2020). The use of three or more anti-viral drugs simultaneously is not recommended (Cascella et al., 2020).

\section{A. Nutrition treatment in the management of COVID-19} virus.

Applicable management strategies for patients with COVID-19 is a rapidly developing therapeutic challenge and the ideal proxies to treat infection or prevent progression to critical illness remain nebulous.Researchers now look for approaches that can reduce the number of severe COVID-19 cases and consequently reduce the mortality rate of the disease. More effective COVID-19containment protocols to ensure shorterhospital stays, less need for prolonged mechanical ventilation, and to reduce death are still missing (https://gisanddata.maps.arcgis.com/apps/opsdashboard/inde x.html\#/bda7594740fd40299423467b48e9ecf6 ( Accessed on 2020-06-10]).

Malnutrition increases mortality, morbidity and causes significant economic impact on the health care systems but also the economic situation of a country influences all aspects of optimal nutrition care (Curtis, 2017). The increased risk of mortality and morbidity caused by malnutrition is a result of the increased rate of infections, as well as delayed recovery. Furthermore, infections increase the demand for several nutrients (Rytter, 2014). It is well-recognized that nutrition is a crucial factor in modulating immune homeostasis. Protein-energy malnutrition or even subclinical deficiencies of one micronutrient may impair one's immune responses (Bhaskaram,2001). Calder et al. 2020 highlighted the importance of optimal nutritional status to protect against a viral infection and $\mathrm{Wu}$ et al. 2020 have provided nutritional advice to reduce damages to the lungs from coronavirus and other lung infections (Wu, 2020).Nutrition treatment in the management is very crucial in enhancing immune response of an infected and vulnerable persons against COVID-19infection (COVID-19 UNICEF Programme 2020) since there are currently, no approved vaccines or pharmaceutical therapies against COVID-19 and identification of specific vaccination strategies and pharmaceutical targets takes long and requires time for research, evaluation and regulatory approval (Derwanda, 2020). Nevertheless,COVID-19 related deaths are in the increase.

Melatonin is a suitable candidate either as antiviral or to treat cytokine storms associated with disease and clotting issues. The initiation of ARDS (Acute respiratory distress syndrome) and ALI (Acute lung injury) is dependent upon the activation of inflammasomes (intergral part of innate immune system). Inflammasomes are able to sense pathogen danger associated molecule patterns (DAMPs) as well as biological crystals including urate and cholesterol. The activation of inflamatones releases proinflammatory cytokines interleukin (IL)-1 $\beta$ and 1L-18. NILRP3 inflamasome is the key to induction of ARDS/ALI. Interleukin 1beta $(1 \mathrm{~L}-1 \beta)$ is a potent proinflamatory cytocine often implicated in the pathogenesis of acute respiratory distress syndrome because the initiation of hypoxemia (low oxygen level in blood) is induced by $1 \mathrm{~L}-1 \beta$ signaling and is tightly controlled and dependent upon NLRP3 inflamasome activation (https://www.ncbi.nlm.gov/pmc/articles/PMC6172mmr-1805-4399)

The fact that pro-inflammatory cytokine storm effects are induced by activation of NLRP3 inflammasomes, the ability of melatonin to inhibit NLRP3 inflamasomes elevates it to a greater position in the fight against COVID-19. Patient with sufficient melatonin regardless of age experience lower infectiousness of COVID-19 with reduced chances of developing ARDS/ALI. Melatonin level is reported to drop with age. Melatonin supplementation dosage is around 0.3 milligram in adults per day (https://pubmed.ncbi.nlm.nih.gov/15649738-melatonin-as-ahypnotic-pro/).

Singapore general hospital researchers recently published a study showing that a combination of easily available and cheap diatary supplements composed of Vitamn D3, B12 and mineral magnesium reduced the progression of COVID-19 to severe or fatal stages (https//medrxiv.org/content/10.1101/2020.06.0). They noted that hyperinflamation played a crucial role in patient outcomes. This meant that direct viral injury was not the only or even major player in organ dysfunction related to COVID-19. Rather, it is the result of the organ toxicity caused by the unregulated release of pro-inflammatory cytokines like IL- 6 and IL- 8 in response to the immune induction by the virus. Hence immunomodulation is an attractive option in the treatment of COVID-19 and in prevention of disease progression. 
(https//medrxiv.org/content/10.1101/2020.06.0).

It is known that vitamin D protects the respiratory epithelium structure and function (https://www.thailandmedical.news/p/sponsorship. The impact of Vitamin D in enhancing immune response against flu and previous coronaviruses has been established. Vitamin D can also suppress cytokine storm, which may substantially increase the chances of avoiding a severe case of COVID-19. Some researchers have suggested a potential association between Vitamin D and CFR during the 1918-1919 viral influenza pandemic, also through suppression of cytokine storm (Daneshkhahet al., 2020).Magnesium promotes vitamin $\mathrm{D}$ functions acting as co-factors in multiple enzymes involved in vitamin D metabolism. It also has independent bronchodilator and vasodilator activities whereas vitamin B12 improves the health of the gut bacteria which in turn is vital for an active and effective immune system. All these supplements have been found to be safe and well tolerated by patients. They have also been more effective in less severe and critical phase of the illness and have proved to reduce the cytokine storm associated with terminal organ damage and death. This could be adopted as safe and easily administered. It is also an essential early intervention in the primary care setting which can effectively be used to prevent symptomatic or severe disease among high risk contact groups traced. It is extremely cost-effective, making it suitable for low and middle income countries even when vaccines and therapeutic drugs may be too costly or unavailable. Significantly, the use of DMB protocol is equally effective in other viral infections that produce high level cytokines causing injury independent of the direct tissue injury (https://www.thailandmedical.news/p/sponsorship

State medical university researchers in Russian had also shown that deficiency of endogenous glutathione especially in older individuals could be one of the contributing factors leading to the progression of the disease from mild infections to severe conditions (http://www.researchgate.net/publication/340917045).

Deficiency of glutathione has been associated with growing number of chronic diseases. As a person ages glutathione levels typically falls while men tend to have lower glutathione levels than females as they age. Glutathione deficiency is also associated with insufficient consumption of fresh vegetables and fruits. It is also an anti-oxidant that demonstrates antiviral properties. It prevents oxidative stress and inflammation and boosts the functions of the immune cells. Glutathione is naturally manufactured in the human body and can only be supplemented in the body via intravenous injections of glutathione whose bioactivity is not easy to procure and maintain. It can also be supplemented with NAC or N-Acetyl cysteine as it is a precursor for glutathione production in the body. NAC significantly inhibits replication of various viruses at different stages of viral life cycle, decreasing viral load and preventing the massive release of inflammatory cells into the lung (cytokine storm). Trial of the antiviral efficiency of such treatment had been done on influenza virus and it significantly reduced the incidence of clinically apparent influenza especially in elderly and high risk individuals (https://www.thailandmedical.news/p/sponsorship

Poor immunity fosters faster progress of COVID-19-19 (Li et al., 2020; Wang et al., 2020). There should be intake of plenty of citrus fruits having various vitamins. Some dry fruits like almonds, walnuts and dates in the improvement of the immune system. However, older people and the patients may take vitamins, iodine and zinc supplements with the consultation of medical practitioners. A decline in immune function is usuallyinevitable with ageing in a process referred to scientificallyas 'immunosenescence'. This usually involvesdeterioration of both innate and acquired immunesystems contributing to decline in $\mathrm{T}$ cell function. Decline in $\mathrm{T}$ cell function is attributed to thymic involution and subsequent reducedoutput of naive T cells. It also brings about ageing-associatedinflammation, often referred to as 'inflammaging', which paradoxically reduces immunity and contributes to pathogenic age-related diseases and poor micronutrient status. This is a bidirectional relationship between infection/immunity and nutrition whereby changes in one of these components can impact the other.Ageing can modulate immune function and cellular composition in ways that are sex-specific, possibly due to differences in how menopause and andropause unfold. Smoking and use of narcotic products is not recommended. Adequate sleep regular exercises and stress free life is essential to boost immune system.

There are several vitamins and trace elements which are essential for the normal functioning of the immune system (Wintergerst et al., 2007). Vitamin A and D supplementation have increased the humeral immunity of paediatric patients following influenza vaccination (Patel,2019). A high dose Zinc supplementation has shown immune enhancement of patients with torquetenovirus (TTV) (Lovino, 2018). Similarly, selenium supplementation has shown a positive response after an influenza vaccination challenge (Ivory, 2017). In addition to micronutrients, several herbal and probiotics have also shown effectiveness for treatment and prevention of viral infections (Mousa, 2017). Moreover, several nutraceuticals and probiotics have indicated supportive role in enhancing immune responses (Mc Carty, 2020).Priority has been given for supplementation of vitamins, trace-elements, nutraceuticals and probiotics.

\section{Medicinal mushrooms against COVID-19 virus}

Medicinal mushrooms have been known to be immune modulators. They contain isolated compounds that result in antiviral protection. Host defense is one of the most recognized brands of mushrooms, specifically, a blend of 17 potent mushroom species. It has been considered one of the most comprehensive immune support formula. Single herb supplements, such as Astragalus, can also be used for a compromised immune system. It is very efficient and may be given as an IV treatment in some hospitals. Compounds in Astragalus are known to increase body's production of white blood

cells

(https://www.ncbi.nlm.nih.gov/pmc/article/PMC4006993).

\section{Vitamin C}


Several reports have shown that vitamin $\mathrm{C}$ could be beneficial in the treatment of pneumonia(Hemila , 2017).Historically, vitamin $C$ has been used for immune support and is known to beacquired by neutrophils (Liugan, 2019). It supports epithelial barrier function against pathogens, cellular functions of the adaptive and innate immune systems, and protects against oxidative stress (Carr, 2017). An earlier reviewundertaken in 2013, found outa reduction inpneumonia incidence in the vitamin $\mathrm{C}$ groups (Hemilia, 2013). From a study, it wasconcluded that therapeutic use of vitamin $\mathrm{C}$ supplementation could be of benefitto patients withpneumonia and that low plasma vitamin $\mathrm{C}$ levels isa health risk. It is well recognized that infections increase oxidative stress (Hamilia, 2017). They activate phagocytes which release reactive oxygen that are oxidising agents. Vitamin $\mathrm{C}$ is a renowned antioxidant which can counteract these effects. In one study a respiratory syncytial virus reducedthe expression of antioxidant enzymes and subsequently increased oxidative damage(Hosakote, 2011). In-vivo animal studies in mice have shown that it is an essential factor for the antiviral immune responses against the influenza A virus (H3N2) through the increased production of interferon- $\alpha / \beta$ especially at the early stages of the infection. There is also enough evidence on the role of vitamin $\mathrm{C}$ in preventing and treating the common cold.Foods that are naturally abundant in vitamin Csuch as broccoli, blackcurrants, fortified breakfast cereals and oranges, red chilli, red capsicum, strawberries, citrus fruits, papaya, mango, kale, watercress, cauliflower, asparagus, okra, lima beans, black-eyed peas, brussel sprouts and spinachare very rich in vitamin C. (Jayawardena, 2020)

\section{Vitamin D}

Vitamin $D_{2}$ also known as ergocalciferol is found in plants while Vitamin D3, cholecalciferol is found in animal tissues. They are converted into active compounds by hydroxylation in the liver and kidneyand therefore they are pharmacologically inactive. Vitamin D is important for bone metabolism and regulates calcium concentrations in the blood. It also increases absorption of calcium from the gut and reduces the amount lost by the kidneys(Jayawardena, 2020). A number of studies have investigated the inter-relationships between vitamin $\mathrm{D}$ and its effects on respiratory viruses and it is well appreciated that vitamin D is a powerful immunoregulator. Vitamin $\mathrm{D}$ receptors being expressed by the majority of immune cells such as B and T lymphocytes, macrophages and monocytes(Sassi etal., 2018). It has been reportedthat respiratory epithelial cells, macrophages and monocytes expressvitamin D receptor and that vitamin D couldact as a potential adjuvant in protecting and treatingpatients with respiratory viral infections who typically have lower vitamin D status (Zdrenghea et al., 2020).It has also beenreported that vitamin D deficiency is associated with a higher risk of viral acute respiratory infection and that its metabolites modulates the expression and secretion of type 1 interferon, chemokines CXCL8 and CXCL10. Proinflammatory cytokines was shown to increase antiviral defences and increase rhinovirus-induced interferon-stimulated genes and cathelicidin, an indication of
a protective
responsetumour
necrosis
factor and

interleukin-6(Telcian etal., 2017).

Vitamin D is predominantly obtained by synthesizing D3 in skin using ultraviolet B light. Small quantities are obtained from food sources. Countries at high latitudes, the ultraviolet $\mathrm{B}$ light in winter is not adequate to synthesize enough Vitamin D. In this case oral intake of the Vitamin D supplement would be important. Dietary sources of Vitamin $\mathrm{D}$ include animal products like oily fish, red meat, liver and egg yolk. Fortified infant formula milk, cereals and margarines are also important. Some mushrooms have also been found to provide Vitamin D if grown under ultra violet light. Other important dietary components include; Vitamin A obtainedfound liver, kidney, butter, egg yolk, deep-sea fish, carrots, dark leafy greens, sweet potato, pumpkin, broccoli, red capsicum, papaya, nectarines and dried apricots. Vitamin $\mathrm{E}$ found in nuts, seeds, whole grains, red meat, prunes, peaches, spinach, broccoli, tomato, asparagus, avocado, potatoes and cabbage and Omega 3fatty acids found in oily fish, flaxseeds, hemp seeds, cod liver oil and walnuts( Jayawardena, 2020)

\section{Zinc}

Zinc is an essential metal being involved in a variety of biological processes due to its function as a cofactor, signaling molecule and structural element. It is involved in the regulation of carbohydrate and lipid metabolism as well as functioning of the reproductive, cardiovascular and nervous system (Anatoly et $a l ., 2020$ ). Zinc is regarded as the 'mother' of immune function. It is an essential trace element which plays an important role in growth, development and the maintenance of immune function.It is a general stimulant of antiviral immunity(Prasad, 2013). Zincmay be obtained from sardines, red meat, oysters, beans, seeds, oats, rye, buckwheat, eggs, ginger root, pecans, brazil nuts, oily fish, parsley, garlic, corn, cauliflower and potato. Zinc deficiency has been associated with an increased susceptibility to infectious diseases, including viral infections. Studies have shown that the zinc status of an individual is a critical factor that can influence immunity against viral infections, with zinc-deficient populations being at increased risk of acquiring infections such as HIV or HCV (Read, 2019). Zinc deficiency may contribute to COVID-19 morbidity and mortality in patientswith severe clinical courses of COVID-19 including the elderly, patients with hypertension, diabetes, coronary heart disease and chronic obstructive lung disease. (Derwand and Scholz, 2020).Zn deficiency also predisposed to impaired immune response to pneumococcal surface protein. It also increased nasal $S$. pneumoniae colonization and severe pneumococcal infection in mice resulting in shorter survival time after infection. Particularly, $\mathrm{Zn}$ deficiency was associated with reduced killing activity of phagocytes in pneumococcal infection.

Zinc plays a role in the regulation of intracellular signalling pathways in adaptive and innate immune cells. Due to the clearly demonstrated role of zinc in immunity and impaired zinc status in ageing, metabolic diseases including diabetes, obesity and cardiovascular diseases is speculated that zinc compounds may be used as an adjunct therapy in COVID-19 treatment for increasing antiviral resistance. Zinc was earlier 
suggested as the potential agent for immune support and prevention of H1N1 influenza the'swine flu'.Moreover, $\mathrm{Zn}$ supplementation was shown to improve ciliary length in bronchial epithelium of $\mathrm{Zn}$-deficient rats.It also increased ciliary beat frequency in vitro. Zinc therefore, may hypothetically ameliorate nCoV-2019-induced dysfunction of mucociliary clearance. Generally, zinc was shown to be essential for respiratory epithelium due to antioxidant and anti-inflammatory activity.It is also able to regulate tight junction proteins ZO-1 and Claudin-1 increasing its barrier functions (Anatolyet al., 2020).

The role of zinc in protection against bronchopulmonary infections, as well as the existing indications of the direct impact of zinc on nCoV-2019 cannot be over emphasized (Anatolyet al., 2020). Zinc is involved in inflammation, elevating inflammatory responses and induction of cell-mediated immunity. Existing data clearly demonstrate that $\mathrm{Zn}$ ions may possess anti-inflammatory effects in pneumonia thus limiting tissue damage and systemic effects (Anatolyet al., 2020).Zinc is a key component of pathogen-eliminating transduction pathways that contribute to neutrophil extracellular trap formation(Gammoh, 2017). Specifically, $\mathrm{Zn} 2+$ cations especially in combination with $\mathrm{Zn}$ ionophore pyrithione were shown to inhibit SARS-coronavirus RNA polymerase (RNA dependent RNA polymerase, RdRp) activity by decreasing its replication. These findings demonstrate that $\mathrm{Zn} 2+$ may be considered as a special antiviral agent in COVID-19 treatment (Anatolyet al., 2020). Zinc is an essential component of antibacterial immunity.Among paediatric populations a review of several studies concluded that zinc supplementation for more than 3 months could be effective in preventing pneumonia in children younger than 5 years of age (Saculchit, 2017). Among the elderly it has beenrecognized that inadequate zinc status impairs immune function, reduces pathogenic resistance and is linked to an increased incidence and duration of pneumonialeading to increased mortality. (Barnett, 2010). Rigorous trials however, are yet to determine the efficacy of zinc supplementation. What remains to be confirmed are the dosages and durations that these would need to be taken to be most beneficial. It is important to determine whether different combinations of micronutrients would be most effective in helping to fight against infection. It has been reported that $\mathrm{Zn}$ supplementation ameliorated the association between nasopharyngeal $S$. pneumoniae carriage and acute lower respiratory infection in children. Patients with better immune response to 23-valent pneumococcal polysaccharide vaccine were characterized by significantly higher serum $\mathrm{Zn}$ levels. However, no effect or serotype-specific effect of $\mathrm{Zn}$ on antibody production in response to polyvalent pneumococcal vaccine was observed (Anatolyet al., 2020).

\section{Selenium}

Selenium is a trace element that has a wide range of pleiotropic effects, ranging from antioxidant effects to anti-inflammatory properties (Rayman, 2012). Low selenium status has been associated with an increased risk of mortality, poor immune function, and cognitive decline. A higher selenium concentration or supplementation has shown antiviral effects. This has been demonstrated in a study by Broome et al. 2004 who evaluated whether an increase in selenium intake (50-100 $\mu \mathrm{g} /$ day) improved immune function in adults (Broome, 2004).A 12-week lasting RCT on healthy adults with sub-optimal selenium concentration $(<110 \mathrm{ng} / \mathrm{ml})$ supplemented with daily capsules of yeast enriched with selenium showed both beneficial and detrimental effects. The immune response to flu vaccine was assessed in selenium supplemented and control groups. Selenium supplementation resulted in a dose-dependent increase in T-cell proliferation. However, positive effects were contrasted by lower granzyme B content of CD8 cells. Furthermore, mucosal flu-specific antibody responses were unaffected by selenium supplementation. Selenium supplementation has also demonstrated effects on the delayed type hypersensitivity (DTH) skin response. Selenium can be obtained from brazil nuts, pecan nuts, sesame seeds, oats, brown rice, tuna, salmon, shellfish, red meat, butter, apple cider vinegar, gelatin, egg yolk, cheese, pecans, hazelnuts, chard, mushrooms and garlic(Jayawardena, 2020).

\section{Copper}

Copper plays a crucial role in immunity by participating in the development and differentiation of immune cells. In-vitro studies have shown that copper demonstrates antiviral properties. Thujaplicin-copper chelates has been shown to inhibit replication of human influenza viruses while intracellular copper regulated the influenza virus life cycle. Turnlund et al. 2014 conducted a study to determine the effect of long-term high copper intake on indices of copper status, oxidant damage and immune function. Their results showed that plasma ceruloplasmin activity, benzylamine oxidase and superoxide dismutase were significantly higher when copper intake was $7.8 \mathrm{mg} /$ day in comparison to $1.6 \mathrm{mg} /$ day, indicating an improvement in antioxidant status. However, the higher copper intake $(7.8 \mathrm{mg}$ /day) significantly reduced the percentage of circulating neutrophils, serum IL-2R and the antibody titer against the Beijing strain of influenza (Turnlund, 2004).

\section{Magnesium}

Magnesium plays an important role in controlling immune function by exerting a marked influence on immunoglobulin synthesis, immune cell adherence, antibody-dependent cytolysis, Immunoglobulin M (IgM) lymphocyte binding, macrophage response to lymphokines and $\mathrm{T}$ helper-B cell adherence (Rupp, 2017). Some in-vitro and in-vivo studies suggested that magnesium played a role in the immune response against viral infections (Chaigne-Delalande, 2013). 
Recommended intakes of selected nutrients to support optimal immune function.

\begin{tabular}{|c|c|c|}
\hline Nutrient & Rationale & Recommendation \\
\hline $\begin{array}{l}\text { Vitamins } \\
\text { and trace } \\
\text { elements }\end{array}$ & $\begin{array}{l}\text { Support the cells and tissues of the immune system. } \\
\text { Deficiencies or suboptimal status in these } \\
\text { micronutrients negatively affect immune function and } \\
\text { can decrease resistance to infections. }\end{array}$ & $\begin{array}{l}\text { A multivitamin and trace element } \\
\text { supplement that supplies the nutrient } \\
\text { requirements for age and gender.For } \\
\text { vitamins and trace elements including } \\
\text { vitamins } \\
\text { A, B6, B12, C, D, E, and folate, and trace } \\
\text { elements including zinc, iron, selenium, } \\
\text { magnesium and copper. This is in addition } \\
\text { to the consumption of a well-balanced } \\
\text { diet. }\end{array}$ \\
\hline Vitamin C & $\begin{array}{l}\text { Doses of _ } 200 \mathrm{mg} / \text { day provide saturating levels in the } \\
\text { blood, and support reduction in the risk, severity and } \\
\text { duration of upper and lower respiratory tract } \\
\text { infections. Requirements for vitamin C increase } \\
\text { during infection. }\end{array}$ & $\begin{array}{l}\text { Daily intake of at least } 200 \mathrm{mg} / \text { day for } \\
\text { healthy individuals. In individuals who are } \\
\text { sick, } 1-2 \mathrm{~g} / \text { day is recommended. }\end{array}$ \\
\hline Vitamin D & $\begin{array}{l}\text { Daily supplementation of vitamin D reduces the risk } \\
\text { of acute respiratory tract infections. }\end{array}$ & Daily intake of 2000 IU/day (50_g/day). \\
\hline Zinc & $\begin{array}{l}\text { Marginal zinc deficiency can impact immunity. Those } \\
\text { deficient in zinc, particularly children, are prone to } \\
\text { increased diarrheal and respiratory morbidity. }\end{array}$ & Daily intake in the range of $8-11 \mathrm{mg} /$ day. \\
\hline $\begin{array}{l}\text { Omega-3 } \\
\text { fatty acids }\end{array}$ & $\begin{array}{l}\text { Omega-3 fatty acids support an effective immune } \\
\text { system and helpsresolve inflammation. }\end{array}$ & $\begin{array}{l}\text { Daily intake of } 250 \mathrm{mg} / \text { day of } \mathrm{EPA}+ \\
\text { DHA. }\end{array}$ \\
\hline
\end{tabular}

Calder, 2020

\section{B. Nutraceuticals supplements}

Nutraceuticals confer physiological benefit or protection against a chronic disease. These products may range from isolated nutrients, herbal products, dietary supplements, genetically engineered designer foods, specific diets, and processed foods, such as cereals, soups and beverages (Kalra, 2003). Some nutraceuticals have shown promising results in enhancing immune function. A very recent study by McCarty et al.2020 reported that certain nutraceuticals may help provide relief to people infected with encapsulated RNA viruses, such as influenza and coronavirus by boosting immune responses (McCarty et al., 2020).

\section{Probiotic supplements}

These are live micro-organisms that confer a health benefit to the host, including on the gastrointestinal tract when administered in adequate amounts (Sanders, 2008). They also stimulate immune response by increasing the antibody production (Kanauchi, 2019). The results of a meta-analysis by Kang et al. 2020 implied that probiotics have a modest effect in common cold reduction (Berggren, 2011).
Lactobacillus and Bifidobacterium strains have been used as treatments that are able to reduce the severity of infection or shorten the duration of infection. A study showed the efficacy of lactobacillus for treatment of respiratory tract infection of viral origin (Berggren, 2011).The study highlighted a significant association between Bifidobacterium and increased immune function and intestinal microbiota in elderly (Jayawardena, 2020).

\section{Multi-nutrients supplements}

As evident in various studies, micronutrient deficiency suppresses immune functions by affecting the T-cell-mediated immune response and adaptive antibody response and leads to dysregulation of the balanced host response (Wintergerst, 2007). Selected vitamins and trace elements support immune function by strengthening epithelial barriers, cellular and humoral immune responses. Supplementations with various combinations of trace-elements and vitamins have shown beneficial effects on the antiviral immune response. A study on low dose supplementation of zinc together with selenium on institutionalized elderly patients, showed an increase on humoral response after vaccination in comparison to the 
control group (Girodon, 1999).Antibody titers after influenza vaccine were higher in groups that received trace elements alone or with vitamins, whereas the vitamins only group had significantly lower antibody titers. The number of patients without respiratory tract infections during the study was higher in groups that received trace elements; zinc sulfate and selenium sulfide(Girodon, 1999). However in another study, neither daily multivitamin-mineral supplementation nor vitamin E (200 mg/day) showed a favorable effect on incidence and severity of acute respiratory tract infections in well-nourished non-institutionalized elderly (Graat et al., 2002).

In addition to micronutrients, obesity has long been associated with higher risks of chronic non-communicable diseases. However, recent evidence suggests that it may also be associated with infectious diseases. Very recent clinical findings of patients with COVID-19 showed that severity of the disease is independently associated with BMI $\geq 28 \mathrm{~kg} . \mathrm{m}-2$ (OR, 5.872; 95\% CI, 1.595 to 21.621; $\mathrm{P}=0.008$ ). Translational data suggested that an alteration in the metabolic profile of $\mathrm{T}$ cells in obese individuals impairs the activation and function of critical adaptive immune cells. A study showed that the mean values of white blood cells, total neutrophil count, monocytes, CD3, CD4 and CD8 lymphocytes were significantly decreased in the group that underwent a weight loss program in comparison to the control group. (Jayawardena, 2020).

\section{DISCUSSION}

The SARS-CoV-2 is the third emerging coronavirus of the last 20 years but the one that has led to an evolving pandemic, a fact that urgently requires the implementation of novel therapeutics. Prevention is a key player in combating pandemics. Nutritional interventions to enhance immunity in viral infections taking into consideration the current epidemic of COVID-19 is of great importance.Evidence of immunityof several vitamins, particularly A, D and E, as well as a few trace elements, such as Zinc and Selenium has been documented. Furthermore, a large number of nutraceuticals and probiotics have shown immune enhancing effects against viral infections, especially influenza-like illnesses.The efficacy and safety of nutraceuticals depend on their ingredients as well as various other factors including methods of extraction(Wintergerst, 2007). A well balanced and varied diet is essential to minimize vitamin deficiencies, but also controlled consumption or supplementation. In fact, high-dosage of vitamin $\mathrm{E}$ supplementation may increase all-cause mortality. Probiotics regulate the functions of systemic and mucosal immune cells and intestinal epithelial cells of the host to regulate immune function, but not all probiotics demonstrate similar health benefits therefore, probiotic products should be carefully selected to get one associated with clinical situation in order to obtain the relevant beneficial effect.Exercise is one of the lifestyle changes that is known to increase immunity and to reduce viral infection.

Everyone including self-quarantine patients should be encouraged to follow food based dietary guidelines.At least five portions of fruit and vegetables each day and all main meals should contain starchy carbohydrate preferably a wholegrain variety. Moreover, two to three portions of meat or equivalent. Vegetarians require pulses and other suitable protein rich foodsincluded on a daily basis. However, taking multi-vitamin-mineral (MVM) supplement for a short period especially during this pandemic would be beneficial since achieving a well-balanced and varied diet is difficult due to several logistics and financial difficulties during lockdowns or self-quarantine. Malnourished or at risk of malnutrition should take extra precautionary care to improve their energy, protein and micronutrient levels.Patients with excess body weight (BMI $>25 \mathrm{~kg} \mathrm{~m}^{-2}$ ) should lose their body weight to improve their immunity. Patients with diabetes mellitus require a varied and balanced diet to maintain blood glucose and enhance immune functions. They should give priority to foods with low glycemic index, limit consumption of high fat and starchy or sugary foods and choose lean protein variety. Supplementation of zinc (20mg/daily) for optimal immune function is required since nearly $1 / 5$ of the world's population is at risk of inadequate zinc intake. Graat etal., 2002).

Regarding nutraceuticals, many single and combined products have shown effectiveness in enhancing immunity in viral infections including influenza. Depending on the availability; many nutraceuticals can be used to enhance immunity. Among the very different important products; garlic, oily fish, cranberry juices and broccoli sprouts are relatively readily available options. Probiotics have been effective for improving the immunity in general and lactobacillus varieties can be recommended to prevent influenza like viral infections (Berggren, 2011).

Every patient who has been diagnosed with COVID-19 must be screened for malnutrition on admission using a validated nutrition screening tool such as NRS-2002. In addition to dietary assessment, they are all required to test for serum vitamin D levels. According to serum vitamin D levels, deficient patients must receive therapeutic doses of vitamin $\mathrm{D}$ according to local guidelines.Some patients may need oral nutrition supplement (ONS) to achieve recommended calories and protein intake. It is also recommended to assess micronutrient deficiencies.

\section{CONCLUSIONS}

There is need to employ correct management stratagems for patients with COVID-19 as it is rapidly evolving. It is of importance that rapid initiation of therapy in high risk populations is coherent and considered. Effective management calls for continual monitoring and adaptation as new literature becomes available. It is therefore critical that institutions involved, work together and report their experiences with the management and treatment of COVID-19 to the medical community for optimization of opportunities.

There are still many open questions that are pending about COVID-19. Although many therapies have been suggested. At present there is none capable of treating COVID-19-19 disease. The only intervention currently viable and proven to decrease the contagion rate seems to be strict quarantine measures for the general population. Specifically designed randomized clinical trials are urgently needed to determine 
the most appropriate evidence-based treatment modality to reduce the spread of this disease and prevent the burden of any future outbreak.Nutritional strategies for enhancing immunity is something to be explored. Selective micronutrient supplementations may be beneficial especially for vulnerable populations such as the elderly.

Conflict of Interest: None declared.

\section{Acknowledgement}

I acknowledge the scientists and clinician who have rushed to understand and mitigate the threat of COVID-19, sharing their view with others. Enabling me to take a step to collect the recent information submitted and published.

\section{Funding source}

No funding source for this work.

\section{REFERENCES}

[1] Agostini, M.L., Andres, E.L., Sims, A.C. (2018). Coronavirus susceptibility to the antiviral Remdesivir (GS-5734) is mediated by the viral polymerase and the proofreading exoribonuclease. Molecular Biology 9 221-218.

[2] Ahmad, S.M.D. (2020). Glocal School of Pharmacy, Glocal University, Mirzapur Pole, Delhi-Yamunotri State Highway 57, Saharanpur, India A Review of COVID-19 (Coronavirus Disease-2019) Diagnosis, Treatments and Prevention

[3] Anatoly, V.S, Lothar R, Olga, P. Ajsuvakova, Michael, A, Viktor A. Gritsenko, Svetlana, I. Alekseenko. Andrey, A., Svistunov,I., Demetrios,P., Demetrios, A., Spandidos, Jan, A.,Aristidis, T., and Alexey, A.T., (2020)Zinc and respiratory tract infections: perspectives for COVID-19 (review)

[4] Barnett, J.B,. Hamer, D.H., Meydani, S.N. (2010). Low zinc status: a new risk factor for pneumonia in the elderly? Nutr Rev 2010; 68:30-7.

[5] Berggren, A. (2011). Randomised, double-blind and placebo-controlled study using new probiotic lactobacilli for strengthening the body immune defence against viral infections. European Journal of Nutrition. 2011; 50 (3):203-210.

[6] Broome, C.S. (2004). An increase in selenium intake improves immune function and poliovirus handling in adults with marginal selenium status. American Journal of Clinical Nutrition. 2004; 80 (1):154-162.

[7] Calder, P.C, Anitra C. Carr, Adrian F. Gombart and Eggersdorfer M (2020). Optimal Nutritional Status for aWell-Functioning Immune System Is an Important Factor to Protect against Viral Infections

[8] Calder P.C.C., Gombart A.F., Eggersdorfer, M. (2020). Optimal nutritional status for a well-functioning immune system is an importan factor to protect against viral infections. Preprints. 2020:2020030199.

[9] Carr, A,. Maggini, S. (2017). Vitamin C and immune function. Nutrients 2017; 9:1211.

[10] Cascella M, Rajnik M, Cuomo A, Dulebohn, S.C, Napoli, R.D. (2020). Features, Evaluation and Treatment Coronavirus (COVID-19). StatPearls Publishing, Treasure Island, FL; 2020.

[11] Chaigne-Delalande, B. (2013). $\mathrm{Mg} 2+$ regulates cytotoxic functions of $\mathrm{NK}$ and CD8 $\mathrm{T}$ cells in chronic EBV infection through NKG2D. Science (New York, N.Y.) 2013; 341(6142):186-191.

[12] COVID-19 UNICEF Programme 2020

[13] Curtis L.J. (2017). Costs of hospital malnutrition. Clinical Nutrition. 2017; 36(5):1391-1396.

[14] Daneshkhah1, A., Eshein1, A., Subramanian1, H., Hemant K. Roy and Backman1, V. (2020). The Role of Vitamin D in SuppressingCytokine Storm in COVID-19 Patients and Associated Mortality

[15] De Luca, C. (2012). Coenzyme Q (10), vitamin E, selenium, and methionine in the treatment of chronic recurrent viral mucocutaneous infections. Nutrition (Burbank, Los Angeles County, Calif.) 2012; 28 (5):509-514

[16] Derwanda, R. and Scholz, M. (2020). Does zinc supplementation enhance the clinical efficacy of chloroquine/ hydroxychloroquine to win todays battle against COVID-19? Medical hypotheseswww.elsevier.com/locate/mehy

[17] Gammoh, N.Z., Rink, L. (2017). Zinc in infection and inflammation. Nutrients 2017; 9:E624.
[18] Gennaro F.D, Pizzol, D, Marotta C.1, Antunes, M., Racalbuto, V, Veronese $\mathbf{N}$ and Smith, L(2020). Coronavirus Diseases (COVID-19) Current Status andFuture Perspectives: A Narrative Review. International environmental research and public health

[19] Girodon, F. (1999). Impact of trace elements and vitamin supplementation on immunity and infections in institutionalized elderly patients: a randomized controlled trial. Arch International Medicine. 1999; 159 (7):748-754.

[20] Graat J.M., Schouten E.G., Kok F.J. (2002). Effect of daily vitamin $\mathrm{E}$ and multivitamin-mineral supplementation on acute respiratory tract infections in elderly Persons. A randomized controlled trial. JAMA 2002; 288(6):715-721

[21] Hafeez, A, Ahmad, S., Siddqui, S.A., Ahmad, M., Mishra, S. (2020). A Review of COVID-19 (Coronavirus Disease-2019) Diagnosis, Treatments and Prevention. Eurasian journal of medicine and oncology

[22] Hemilä H. (2017). Vitamin C and infections. Nutrients 2017; 9:339.

[23] Hosakote, Y.M., Jantzi, P.D., Esham, D.L., (2011). Viral-mediated Inhibition of antioxidant enzymes contributes to the pathogenesis of severe respiratory syncytial virus bronchiolitis. Am J Respir Crit Care Med 2011; 183:1550-60

[24] https://www.who.int/bulletin/online_first/20-260281.pdf

[25] https://www.ncbi.nlm.gov/pmc/articles/PMC6172mmr-18-05-4399) https://link.springer.com/article/10.1186/s12974-017-1051

[26] https://pubmed.ncbi.nlm.nih.gov/15649738-melatonin-as-a-hypnotic-p ro/

[27] https://www.thailandmedical.news/p/sponsorship

[28] https://gisanddata.maps.arcgis.com/apps/opsdashboard/index.html\#/bd a7594740fd40299423467b48e9ecf6

[29] https://www.ncbi.nlm.nih.gov/pmc/article/PMC4006993

[30] Huang, C., Wang, Y., Li, X., Ren, L., Zhao, J., Hu, Y. 2020. Clinical features of patients infected with 2019 novel coronavirus in Wuhan, China. Lancet 395 (10223), 497-506.

[31] Hui, D.S., IA, E.,Madani, T.A., Ntoumi, F., Kock, R., Dar, O. 2020 The continuing 2019-nCoV epidemic threat of novel coronaviruses to global health - the latest 2019 novel coronavirus outbreak in Wuhan, China. International. Journal of Infectious. Diseases. 91, 264-266.

[32] Ivory K. (2017). Selenium supplementation has beneficial and detrimental effects on immunity to influenza vaccine in older adults. Clinical Nutrition. 2017; 36(2):407-415.

[33] Jayawardena, R, Sooriyaarachchi, P, Chourdakis,M Jeewandara, C, and Ranasinghe, P. (2020).Enhancing immunity in viral infections, with special emphasis on COVID-19: A review

[34] Jaimes, J.A.,Millet, J.K., Stout, A.E., Andre, N.M.,Whittaker, G.R. (2020). A tale of two viruses:the distinct spike glycoproteins of feline coronaviruses. Viruses 12, 83.

[35] Kalra E.K. (2003). Nutraceutical definition and introduction. AAPS Pharmacutical Sciences. 2003; 5(3) E25-E25.

[36] Kang E.J. (2013). The effect of probiotics on prevention of common cold: a meta-analysis of randomized controlled trial studies. Korean journal of family medicine. 2013; 34(1):2-10.Kenya, clinical nutrition and dietetics reference manual. 2020.

[37] Li, Q., Guan, X., Wu, P., Wang, X., Zhou, L., Tong, Y. (2020). Early transmission dynamics in wuhan, China, of novel coronavirus-infected pneumonia. New England Journal of Medicine. 382, 1199-1207.

[38] Liugan, M., Carr, A.C. (2019). Vitamin C. Vitamin C and neutrophil function findings from randomized controlled trials. Nutrients 2019; 11:2102.

[39] Lu, R., Zhao, X., Li, J., Niu, P., Yang, B., Wu, H., 2020. Genomic characterization and epidemiology of 2019 novel coronavirus: implications for virus origins and receptor binding. Lancet 395 (10224), 565-574.

[40] Miguel Angel Martinez. (2020).Compounds with therapeutic potential against novel respiratory coronavirus. Antimicrobial Agents and Chemotherapy, 2020; DOI: 10.1128/AAC.00399-20

[41] Kenya, clinical nutrition and dietetics reference manual

[42] Mousa, H.A. (2017). Prevention and treatment of influenza, influenza-like illness, and common cold by herbal, complementary, and natural therapies. J Evid Based Complementary Altern Med. 2017; 22(1):166-174.

[43] McCarty, M.F., DiNicolantonio, J.J. (2020). Nutraceuticals have potential for boosting the type 1 interferon response to RNA viruses including influenza and coronavirus. Progressive Cardiovascular Disease.

[44] Patel, N. (2019). Baseline serum vitamin A and D levels determine benefit of oral vitamin A and D supplements to humoral immune 
responses following pediatric influenza vaccination. Viruses. 2019; 11 (10).

[45] Prasad, A.S. (2013).Discovery of human zinc deficiency: its impact on human health and disease. Advances in nutrition. 2013; 4:176-190. doi: 10.3945/an.112.003210. Bethesda, Md.

[46] Rayman, M.P. (2012). Selenium and human health. Lancet. 2012; 379(9822): 1256-1268.

[47] Ren, L.L., Wang, Y.M., Wu, Z.Q., Xiang, Z.C., Guo, L., Xu, T. 2020. Identification of a novel coronavirus causing severe pneumonia in human: a descriptive study. Chinese Medical Journal.

[48] Read, S. A., Obeid, S., Ahlenstiel, C and Ahlenstiel, G. (2019). The role of zinc in antiviral immunity. Advances in Nutrition, 10(4), 696-710.

[49] Rytter M.J. (2014). The immune system in children with malnutrition--a systematic review. PloS One. 2014; 9(8) 10 Bhaskaram P. Immuno-biology of mild micronutrient deficiencies. Br J Nutr. 2001; 85(Suppl 2):S75-S80.

[50] Rupp, J.C. (2017). Host cell copper transporters CTR1 and ATP7A are important for influenza a virus replication. Virology Journal. 2017; 14(1):11.

[51] Sheahan, T.P., Sims, A.C., Leist, S.R. (2020). Comparative therapeutic efficacy of remdesivir and combination lopinavir, ritonavir, and interferon beta against MERS-CoV. Nat.Commun. 11, 222.

[52] Telcian, A.G,. Zdrenghea, M.T., Edwards, M.R, (2017). Vitamin D increases the antiviral activity of bronchial epithelial cells in vitro. Antiviral Rev 2017:137:93-101

[53] Turnlund, J.R. (2004). Long-term high copper intake: effects on indexes of copper status, antioxidant status, and immune function in young men. American Journal of Clinical Nutrition. 2004; 79 (6):1037-1044.

[54] Wan, Y., Shang, J., Graham, R., Baric, R.S., Li, F., (2020). Receptor recognition by novel coronavirus from Wuhan: an analysis based on decade-long structural studies of SARS.Journal of Virology. 94, 31996437.

[55] Wintergerst E.S., Maggini S., Hornig D.H. (2007). Contribution of selected vitamins and trace elements to immune function. Annual of nutritional Metabolism. 2007; 51(4):301-323.

[56] Wang,Y., Wang, Y., Chen, Y., Qin, Q. (2020).Unique epidemiological and clinical features of the emerging 2019 novel coronavirus pneumonia (COVID-19) implicate special control measures [Epub ahead of print]. Journal of Medical Virology. 2020, 10.1002/jmv. 25748

[57] WHO. (2020).Coronavirus disease (COVID-19) Situation Report 126.

https://www.who.int/docs/default-source/coronaviruse/situation-report s/20200525-COVID-19-sitrep-126.pdf?sfvrsn=887dbd66_2

[58] Wu, J.Z., P. (2020). Treatment strategies for reducing damages to lungs in patients with coronavirus and other infections. .Preprints; $p$. 2020020116

[59] Wintergerst E.S., Maggini S., Hornig, D.H. (2007). Contribution of selected vitamins and trace elements to immune function. Annual Nutrition Metabolism. 2007; 51(4):301-323.

[60] Zhou, F., Yu,T., Du, R, (2020). Clinical course and risk factors for mortality of adult inpatients with COVID-19 in Wuhan, China: a retrospective cohort study. Lancet 2020; 395(10229):1054-62. 